\title{
All-Particle Multiscale Computation of Hypersonic Rarefied Flow
}

\author{
Eunji Jun*, Jonathan M. Burt ${ }^{\dagger}$ and Iain D. Boyd ${ }^{*}$ \\ Department of Aerospace Engineering, University of Michigan, Ann Arbor, MI 48109
}

\begin{abstract}
Hypersonic aerothermodynamics for a probe entering a planetary atmosphere is an important issue in space exploration. The probe experiences various Knudsen number regimes, ranging from rarefied to continuum, due to density variation in the planet's atmosphere. To simulate such multiscale flows, a novel hybrid particle scheme is employed in the present work. The hybrid particle scheme employs the direct simulation Monte Carlo (DSMC) method in rarefied flow regions and the low diffusion (LD) particle method in continuum flow regions. Numerical procedures in the low diffusion particle method are implemented within an existing DSMC algorithm. The hybrid scheme is assessed by studying Mach 10 nitrogen flow over a sphere with a global Knudsen number of 0.002. Using the same mesh, the hybrid scheme results show good overall agreement with results from standard DSMC computation. Subcell procedures are utilized to improve computational efficiency and reduce sensitivity to DSMC cell size in the hybrid scheme. The LD-DSMC approach is also assessed by comparing hybrid results obtained using different cell sizes.
\end{abstract}

\section{Nomenclature}

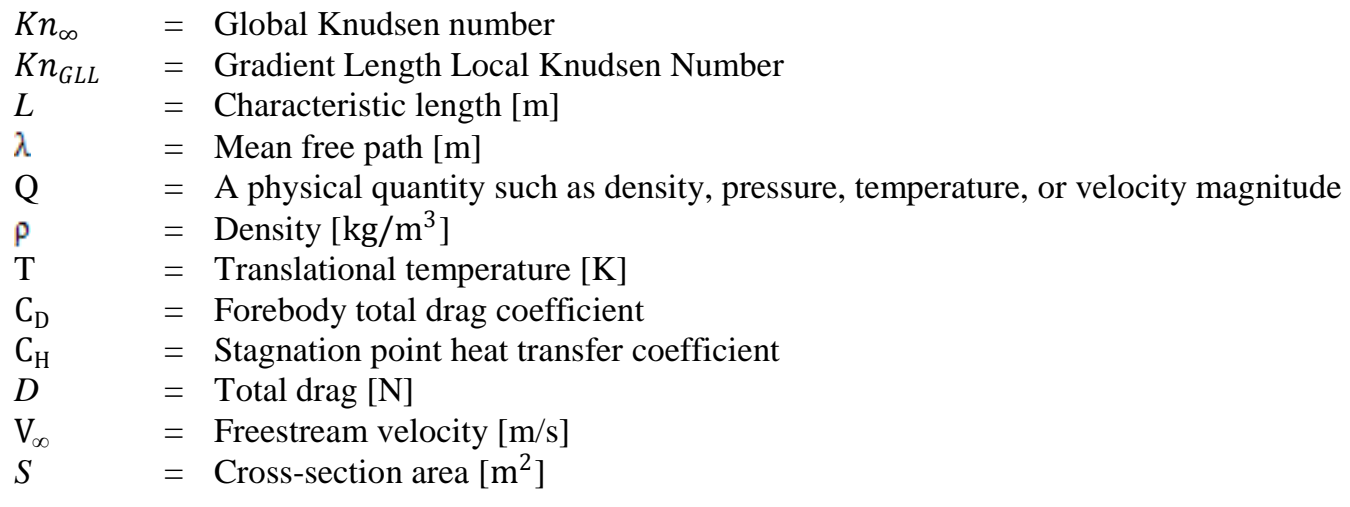

\section{Introduction}

$\mathrm{T}$ HE desire of humans for space exploration was first realized through the success of Sputnik in 1957 and over 200 missions have been completed or are planned since that time. As we move into the $21^{\text {st }}$ century, planetary missions, especially to the Moon and Mars, have taken center stage in planetary exploration due to the possibility of finding water and perhaps life. ${ }^{1}$ To explore the surface of a planet possessing an atmosphere, most planetary missions utilize a hypersonic entry capsule. For design and development of the hypersonic vehicle, it is necessary to evaluate the performance during entry into a planet's atmosphere. Experiments and flight tests are both extremely challenging and expensive, so there is a need for accurate and efficient computational aerothermodynamics models. A hypersonic entry vehicle will experience many different flow regimes due to the change in atmosphere density with altitude. For example, Mars Pathfinder directly entered the Mars atmosphere as an entry capsule. ${ }^{2,3}$ It was released from the spacecraft at $130.9 \mathrm{~km}$ and began a descent to the surface during which, it experienced a wide range of flow regimes, including both high and low Knudsen number regions, as shown in Table 1.

\footnotetext{
* Graduate Student, Member AIAA

${ }^{\dagger}$ Post-doctoral research fellow, Member AIAA

* Professor, Associate Fellow AIAA
}

American Institute of Aeronautics and Astronautics 
Table 1. Freestream and surface conditions for Mars pathfinder nominal entry trajectory ${ }^{2,3}$

\begin{tabular}{ccccc}
\hline Altitude $[\mathbf{k m}]$ & Time $[\mathbf{s}]$ & $\mathbf{V}_{\infty}[\mathbf{m} / \mathbf{s}]$ & $\boldsymbol{\rho}_{\infty}\left[\mathbf{k g} / \mathbf{m}^{\mathbf{3}}\right]$ & $\mathbf{K n _ { \infty } , \mathbf { H S }}$ \\
\hline $\mathbf{1 3 0 . 9}$ & 0.0 & 7463.1 & $1.1767 \times 10^{-9}$ & $2.41 \times 10^{1}$ \\
$\mathbf{1 1 0 . 1}$ & 12.1 & $7,472.8$ & $1.8386 \times 10^{-8}$ & $1.54 \times 10^{0}$ \\
$\mathbf{9 0 . 2}$ & 24.6 & $7,481.5$ & $2.6074 \times 10^{-7}$ & $1.09 \times 10^{-1}$ \\
$\mathbf{6 5}$ & 42.3 & $7,453.9$ & $7.9450 \times 10^{-6}$ & $3.57 \times 10^{-3}$ \\
$\mathbf{5 6 . 1}$ & 49.3 & $7,431.3$ & $2.7614 \times 10^{-5}$ & $1.03 \times 10^{-3}$ \\
\hline
\end{tabular}

In assessing departure from translational thermal equilibrium, a flow can be characterized by the overall Knudsen number as given in Eq. (1).

$$
\mathrm{Kn}=\frac{\lambda}{\mathrm{L}}
$$

where $\lambda$ is the mean free path of the gas and $\mathrm{L}$ is a characteristic length scale. The flow is considered to be continuum if the Knudsen number is much smaller than one. In this case, one can disregard microscopic structure in the gas and consider only macroscopic parameters such as density, velocity, and temperature. For a Knudsen number tending to infinity, the flow regime can be considered free-molecular. In this case, particle collisions with the body surface play the determining role in forcing the gas velocity distribution away from equilibrium. In the transitional regime between free-molecular and continuum regimes, both gas-surface collisions and intermolecular collisions are important. If the Knudsen number is much smaller than 1, then a sufficiently large number of collisions occur for the velocity distribution to be characterized by a small departure from equilibrium, and the conventional Navier-Stokes or Euler equations are appropriate models. As the Knudsen number increases, however, the continuum assumption breaks down due to insufficient collisions, and the Navier-Stokes equations become inaccurate. At this high Knudsen number, the flow is characterized by strong thermal non-equilibrium and requires a simulation method based on kinetic theory. Many flows include both high Knudsen number and low Knudsen number regions; typical examples are high-speed flow (atmospheric flow around reentry vehicles and other hypersonic vehicle), and low density flow (rocket exhaust plumes at high altitude). The flow regimes and characteristics are explained in Figure 1. ${ }^{4}$ The Euler or Navier-Stokes equations are valid for the low Knudsen number regimes, and continuum computational fluid dynamics (CFD) techniques may be employed to solve those equations numerically. On the other hand, kinetic simulation techniques base on the Boltzmann equation (derived from gas kinetic theory) are valid in both low and high Knudsen number regimes. The velocity distribution is determined by numerically solving the Boltzmann equation or applying a Monte Carlo method, and large deviations from an equilibrium distribution are allowed. However, these methods are very expensive to apply in the low Knudsen number regime. Therefore, it is attractive to develop a hybrid method to analyze multiscale flows involving a wide range of Knudsen number regimes. 5

In recent efforts to develop hybrid schemes for simulating such multiscale flows, the most widely investigated type of method is the CFD-DSMC hybrid scheme. In this hybrid scheme, CFD is used in the near-equilibrium regions and the direct simulation Monte Carlo (DSMC) method is applied in non-equilibrium regions where the continuum Navier-Stokes equations fail. ${ }^{6,7}$ Among hybrid CFD-DSMC implementations, there are two main types of approaches. In an uncoupled approach, CFD calculations are applied only over near-equilibrium regions, and results from this computation are used to define inflow boundary conditions for a DSMC simulation. The coupled approach is more complex and involves a number of challenges. It must integrate two very different methods (CFD and DSMC) into a single numerical framework. In addition, two-way coupled information transfer between CFD and DSMC domains is complex and requires significant algorithm development.

In yet another type of hybrid approach for multiscale flow simulation, DSMC type particles are employed throughout the simulation domain. This approach, which has been termed an "all-particle" hybrid scheme, uses particle methods in place of CFD which are based on DSMC and are intended for efficient simulation of low Knudsen number flows. ${ }^{8,9}$ As in DSMC, these continuum particle methods use particles to transfer mass, momentum and energy through a computational grid, and employ temporal decoupling between particle movement and velocity re-sampling procedures during each simulation time step. Macroscopic flow properties are calculated in both rarefied and continuum regions by averaging quantities among representative particles. Particle velocities are typically redistributed under an assumption of local equilibrium within continuum regions. This method can allow for very strong coupling between the two flow regimes. Among other advantages relative to hybrid CFD-DSMC schemes, this approach allows simpler code development, as there is no need to integrate two very different simulation schemes in the same code. However, "all-particle" hybrid techniques are prone to large errors associated 
with numerical diffusion resulting from free-molecular fluxes between adjacent cells. This numerical diffusion is due to an extreme sensitivity to computational cell size in the equilibrium particle method. Ideally, random molecular motion in the continuum flows should be suppressed on length scales comparable to the cell size because the ratio of local mean free path to cell size is typically very small. Over these macroscopic length scales, individual molecules tend to move along smooth trajectories that approximately follow the gas streamlines. In contrast, representative particles in DSMC-based continuum particle methods exhibit random motion on length scales comparable to the cell size, so that the cell size takes on properties of the local mean free path and becomes a scaling factor for numerical transport coefficients. Because the influence of collisions is neglected in fluxes based on particle motion between adjacent cells, the ratio of numerical transport coefficients (viscosity, thermal conductivity and mass diffusivity) to physical transport coefficients tends to scale with the ratio of the mean free path to the cell size. As a result, both numerical viscosity and thermal conductivity become extremely large when the cell size is much greater that the local mean free path. To avoid this problem, a small cell size is needed, but a prohibitively large number of cells may be required to reach an overall level of simulation accuracy comparable to that of CFD methods on a much coarser gird.

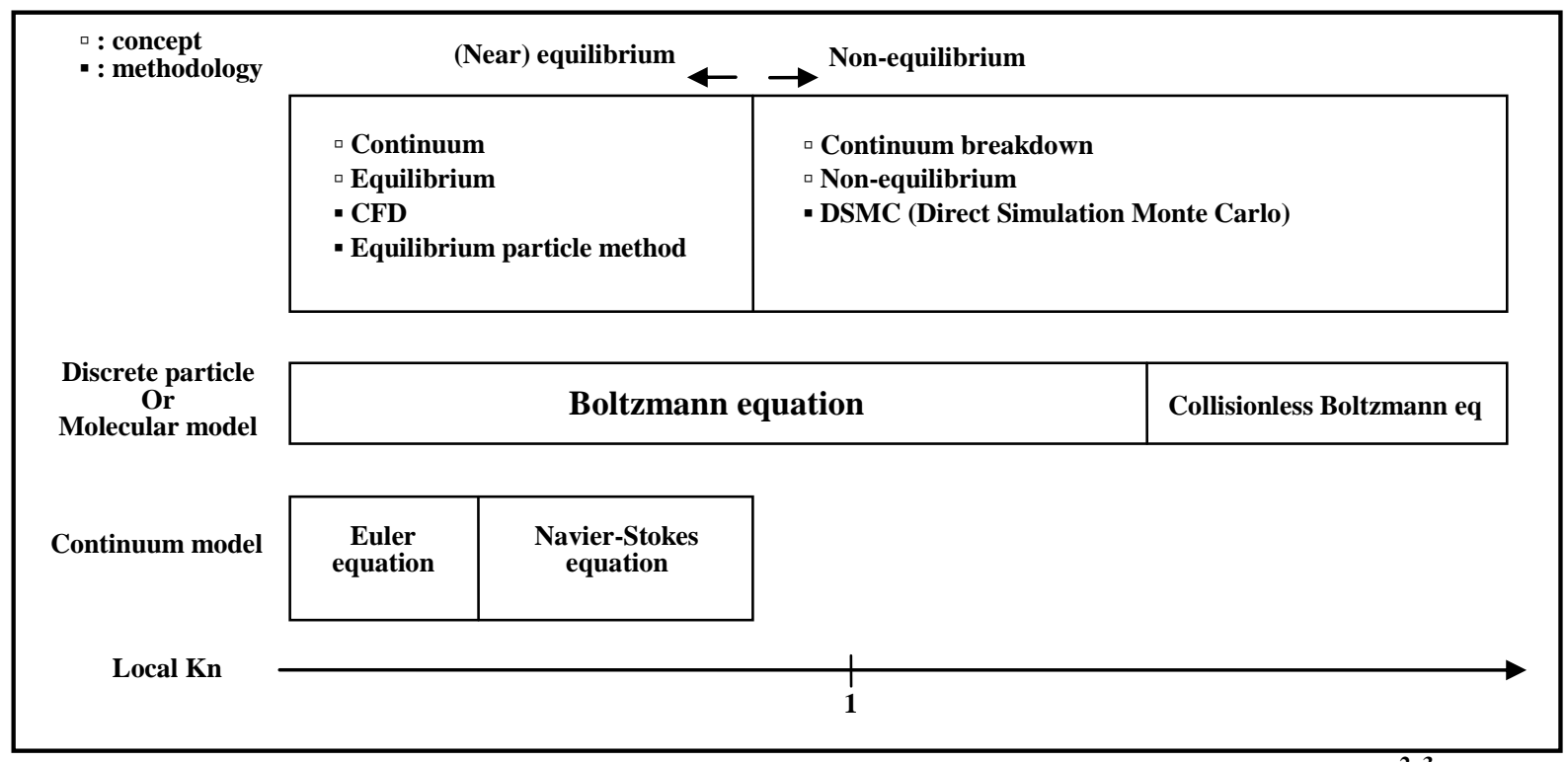

Figure 1. Flow regimes and characteristics according to the local Knudsen number ${ }^{2,3}$

In the present study, a new continuum particle method ${ }^{10-12}$ is used as an alternative means of low Knudsen number gas flow simulation. In this method, originally intended for simulating gas flows near the low Knudsen number limit, a large number of representative particles are tracked through the grid in such a way that every particle maintains a constant relative position within a network of Lagrangian cells. Each Lagrangian cell is coincident with a cell in the fixed Eulerian grid at the beginning of each time step, and moves and deforms over the time step interval according to local bulk gas properties. Particles follow the macroscopic motion of Lagrangian cells, and move along trajectories that closely approximate the gas streamlines. Random particle motion associated with thermal energy is therefore greatly suppressed. As a result, numerical diffusion errors, as well as effects of statistical scatter, are generally far smaller than in other equilibrium particle methods. The new method described here significantly reduces both numerical diffusion effects and statistical scatter relative to existing DSMC-based continuum particle methods, and is therefore termed the low diffusion (LD) method.

This paper will discuss numerical procedures in the LD method and the physical models used in LD method simulations. To assess the validity of an LD-DSMC hybrid scheme, a Mach 10 flow of nitrogen over a 12-inch diameter sphere is considered. Simulations are performed with standard DSMC and the LD-DSMC hybrid method using the same mesh. Previous hybrid LD-DSMC simulations of hypersonic flow ${ }^{12,13}$ did not show efficiency improvements over standard DSMC simulations due to the use of very small cells, along with a very small time step, in continuum regions. However, larger cell and time step intervals can be used within continuum regions assigned to the LD method domain in a hybrid LD-DSMC simulation, as it is not necessary in these regions to satisfy the conventional DSMC guidelines of cell sizes smaller than the mean free path and time step smaller than the mean collision time. To improve computational efficiency in the present work, a subcell utility is employed to allow for a 
coarser mesh in hybrid simulations of a given flow. The paper concludes with a summary of the present findings and some ideas for future work.

\section{Numerical Method and Physical Models}

As mentioned above, the primary merits of the LD method are: 1) greatly reduced numerical diffusion effects and scatter compared with other DSMC-based methods for continuum flow simulation; 2) simple implementation in a hybrid code with DSMC; 3 ) inclusion of physical diffusion effects, for transport coefficients which are relatively independent of cell size; along with 4) capabilities for efficient application of various DSMC models to low Knudsen number flows. In general, the LD method is similar to the DSMC technique. A given computation domain is divided into a large number of computation cells. During each time step, particles are moved through the grid according to their assigned velocities and bulk flow properties are calculated by averaging quantities over all particles in each cell. The main difference between the LD method and DSMC is related to how the velocities used for particle movement are updated during each time step. The LD method starts from the calculation of cell bulk velocity, mass density and the thermal speed for each cell. These values are evaluated based on cell-averaged particle values, and are stored in the cell data structure. Once these quantities have been determined for all cells during the current time step, a series of Lagrangian face velocities are computed. Lagrangian cells are superimposed over fixed Eulerian cells, with identical face locations at the beginning of the timestep. No net momentum or energy can be transferred to the gas from a Lagrangian cell face, but both momentum and energy may be exchanged through this face between two neighboring cells. To account for momentum and energy exchange through Lagrangian faces, bulk velocity and temperature values are updated for all particles during each time step. Then, all particles in each cell are assigned the cell-based bulk velocity and temperature. Finally, the position of each Lagrangian cell vertex at the end of the time step interval is calculated and the velocity used for particle movement is updated. Numerical procedures in the LD method are described in detail in Ref. 10.

To employ both LD and DSMC methods as part of a hybrid scheme, it is necessary to determine a continuum breakdown parameter that assigns appropriate allocation of cells to LD and DSMC domains. Boyd et al. proposed ${ }^{14}$ the use of the gradient-length local (GLL) Knudsen number:

$$
\mathrm{Kn}_{\mathrm{GLL}}=\frac{\lambda}{\mathrm{Q}}\left|\frac{\mathrm{dQ}}{\mathrm{dl}}\right|
$$

where Q is some flow quantity of interest, such as density, pressure, temperature, or velocity magnitude. In this work, LD-DSMC domain decomposition is determined based on the following GLL Knudsen number:

$$
\mathrm{Kn}_{\mathrm{GLL}, \max }=\max \left\langle\frac{\lambda}{\rho}|\nabla \rho|, \frac{\lambda}{\mathrm{T}}|\nabla \mathrm{T}|, \frac{\lambda}{\mathrm{a}}|\nabla \mathrm{u}|\right\rangle
$$

where $\lambda$ is again the mean free path, $p$ is the density, $T$ is the translational temperature, $u$ is bulk velocity magnitude, and $\mathrm{a}$ is the local speed of sound. Regions for which $\mathrm{Kn}_{\mathrm{GLL}, \max }$ is larger than 0.05 are assigned to the DSMC domain, and all other regions are assigned to LD. Domain decomposition is performed once every few thousand time steps.

For both standard DSMC and LD-DSMC simulations, the same physical models are employed. Molecular collisions are simulated using the variable hard sphere (VHS) ${ }^{4}$ model with a reference temperature of $273 \mathrm{~K}$ and a viscosity temperature exponent of 0.75 . Energy exchange between kinetic and internal modes is controlled by the Larsen-Borgnakke statistical model. ${ }^{15}$ The current solutions are obtained without chemical reactions.

\section{Simulation Cases}

Nitrogen flow over a 12 inch diameter sphere at Mach number 10 is studied. The free stream temperature is $200 \mathrm{~K}$ giving a free stream velocity of $2883.5 \mathrm{~m} / \mathrm{s}$. The surface of the sphere has a fixed temperature of $500 \mathrm{~K}$. The mass density, number density and mean free path are given in Table 2. The Knudsen number is calculated using the sphere diameter as the characteristic length and the hard sphere model to determine the mean free path. ${ }^{16}$

Table 2. Simulation flow properties ${ }^{16}$

\begin{tabular}{cccc}
\hline $\mathbf{K n}_{\infty}$ & $\begin{array}{c}\text { Mass Density } \\
\left(\mathbf{k g} / \mathbf{m}^{\mathbf{3}}\right)\end{array}$ & $\begin{array}{c}\text { Number Density } \\
\left(\text { particles } / \mathbf{m}^{3}\right)\end{array}$ & $\begin{array}{c}\text { Mean Free Path } \\
(\mathbf{m})\end{array}$ \\
\hline $\mathbf{0 . 0 0 2}$ & $9.875 \times 10^{-5}$ & $2.214 \times 10^{21}$ & $6.096 \times 10^{-4}$ \\
\hline
\end{tabular}


For the LD-DSMC hybrid scheme, a hybrid mesh is used in which a structured grid is employed along the fore body surface while an unstructured grid is used elsewhere in the flow field (Figure 2). For the structured grid, cell stretching is employed to provide enough particles per cell near the stagnation point. This is a useful technique in axisymmetric simulations, where it is often difficult to achieve a sufficiently large enough number of particles per cell near the axis.
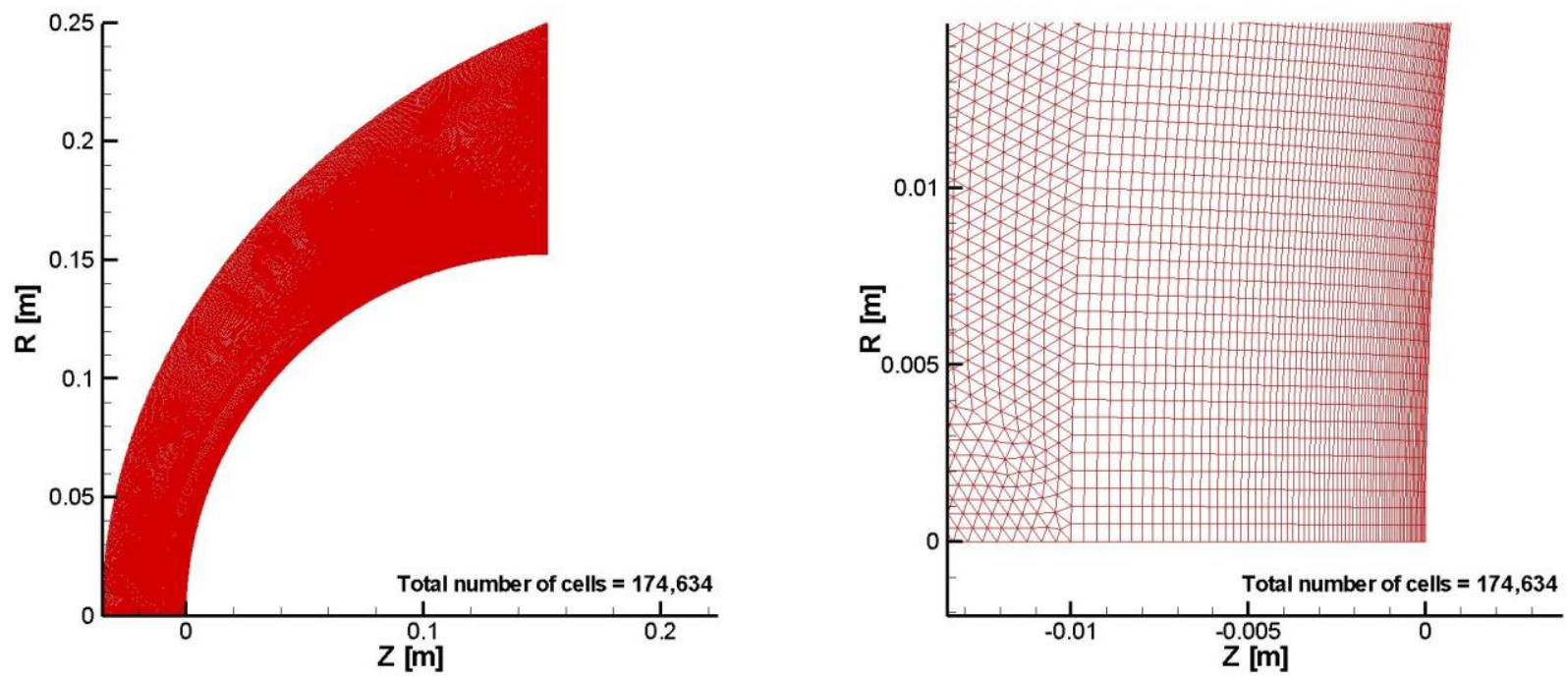

Figure 2. Grid configuration for sphere fore-body

Three simulations are performed: (1) a standard DSMC simulation with 174,634 total cells; (2) a LD-DSMC simulation with the same 174,634 total cells; and (3) a LD-DSMC simulation with 91,342 total cells. Cases (1) and (2) are compared to assess the accuracy of the proposed LD-DSMC method, and cases (2) and (3) are compared to assess the subcell utility that aims to improve the computation efficiency of the hybrid computation.

\section{Results and Discussion}

The purpose of the present work is to validate the LD-DSMC hybrid method. This is achieved by comparing LDDSMC solutions with standard DSMC results. At simulation startup, the entire flow field is assigned to the DSMC domain. A continuum breakdown parameter based on density, temperature and velocity gradients (Equation 3) is then periodically evaluated in all cells within the computational grid. Breakdown parameter values are compared with a cutoff value ( 0.05 is used here) to determine whether each cell should be assigned to DSMC or LD domains; when the parameter value is larger than 0.05 in a given cell, continuum breakdown is assumed and the cell is assigned to the DSMC domain.

Figure 3 (left) shows the LD-DSMC domain decomposition for a $\mathrm{Kn}=0.01$, fore-body sphere case. At this relatively high Knudsen number, most of the computational domain is simulated by the DSMC method and the LD region is confined to a thin layer downstream of the shock. This is a flow that is best computed fully using DSMC. To see potential advantages of the LD-DSMC hybrid simulation, a lower Knudsen number case is more appropriate. Figure 3 (right) shows contours of $\mathrm{Kn}_{\mathrm{GLL}, \max }$ and the method domain boundaries for the case where $\mathrm{Kn}=0.002$. Here, the DSMC regions are confined to the shock wave and the boundary layer due to steep flow field gradients. Two layers of overlapping cells are employed along the boundary between DSMC and LD domains, and are designated as buffer regions A and B. The relative locations of these buffer regions are shown in Figure 4. In buffer region A, adjacent to the DSMC domain, all simulation procedures are carried out as in DSMC cells, while in buffer region $\mathrm{B}$, adjacent to the $\mathrm{LD}$ domain, $\mathrm{LD}$ calculations are performed. This provides a simple and effective means of strongly coupled information transfer between LD and DSMC domains.

Figure 5 (left) shows the stream lines, Mach number and the LD/DSMC domain boundaries for the Kn $=0.002$ case. The upper half is simulated by the standard DSMC scheme and the lower half is simulated by the LD-DSMC hybrid method using the same mesh. In the hybrid method, the detached bow shock is simulated in the DSMC domain, and the boundary layer near the sphere surface is also assigned to DSMC. The freestream and high density 

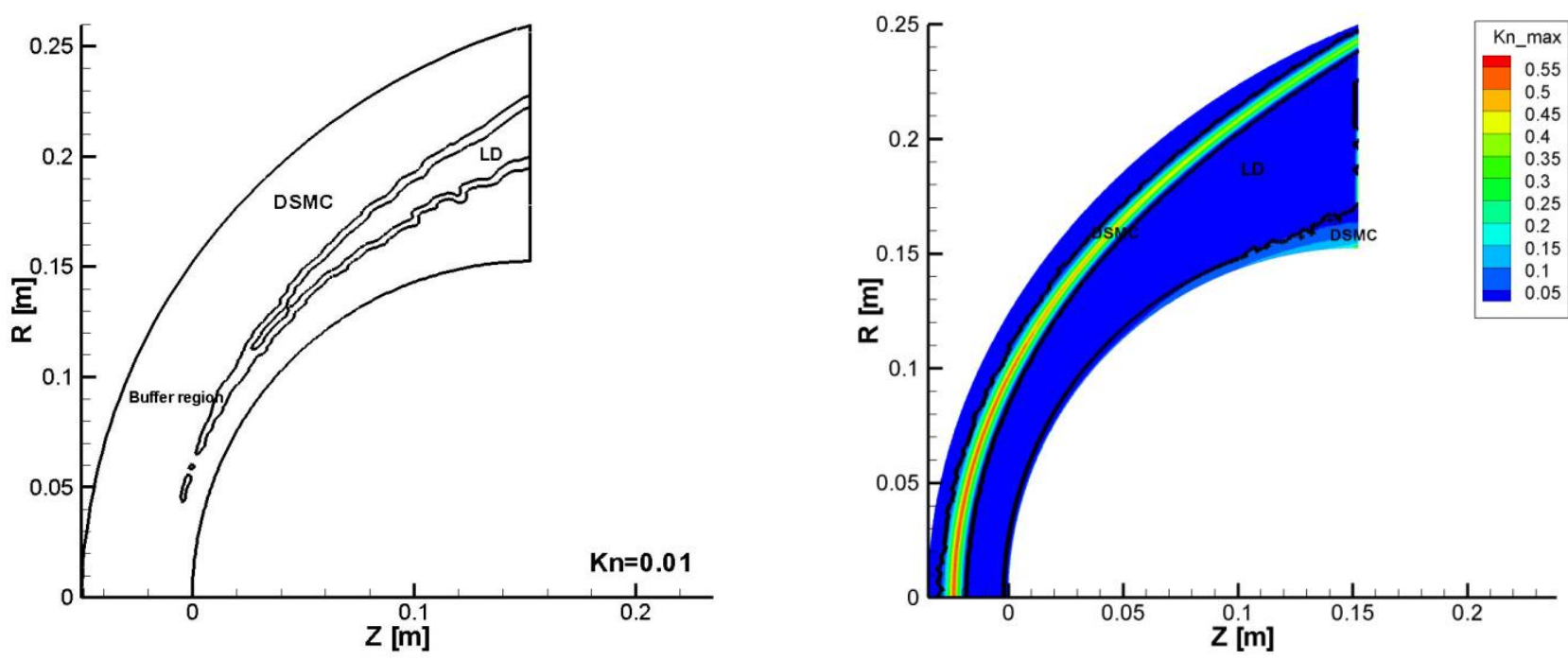

Figure 3. Continuum breakdown domain boundaries

(Left: $\mathrm{Kn}=0.01$, Right $\mathrm{Kn}=\mathbf{0 . 0 0 2}$ )

post shock regions are assigned to the LD domain. Very good agreement is observed between standard DSMC and LD-DSMC hybrid results. For example, the shock standoff distance and shock shapes are nearly identical in the two solutions. The results in Figure 5 (right) are discussed later

Pressure contours from both simulations are shown in Figure 6. The contours represent results from the DSMC simulation, while solid lines give results from the LD-DSMC hybrid simulation using the same mesh. Throughout the entire computational domain, the two simulations are nearly indistinguishable within the resolution of the figure.

Contours of translational and rotational temperature are shown in Figure 7. Rotational temperature is presented to demonstrate the presence of rotational-translational nonequilibrium. The level of agreement in the temperature solutions obtained with the two methods is generally within $2 \%$ and reduced discrepancies are observed away from the stagnation streamline. In general, the use of a cutoff value of the continuum breakdown parameter of 0.05 aims to provide agreement between DSMC and the Navier-Stokes equations within 5\%, so the level of agreement achieved here is considered more than acceptable. Profiles of the temperature along the stagnation streamline are shown in Figure 8. The vertical solid line denotes the boundaries of the LD and DSMC regions. As expected, the DSMC domain includes high gradient regions within the shock and near the sphere surface. Excellent agreement is obtained between the two simulation approaches.

Figure 9 shows profiles of bulk velocity and mass density along the stagnation streamline. In these results, some disagreement is observed between the bulk velocity profiles from the two simulations near the downstream edge of the shock, with a particularly large discrepancy in the post-shock LD region. These differences may be related to viscous transport calculations in the LD method, and could potentially be reduced by either reducing the cutoff value for continuum breakdown in equation (3) or by extending the DSMC domain by a larger number of cells during the cell assignment procedures in the hybrid simulation. Figure 10left shows profiles of translational and

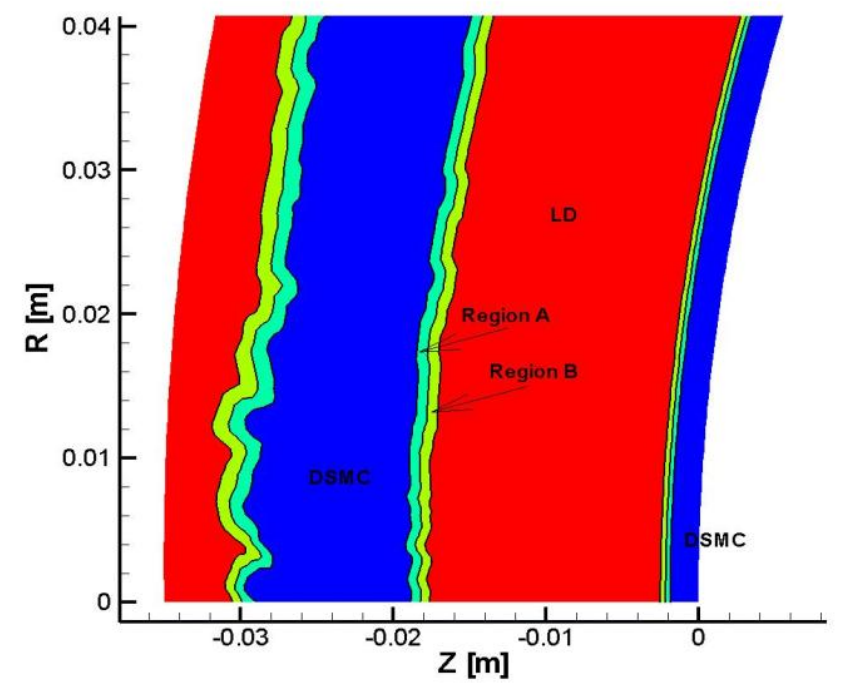

Figure 4. Location of buffer regions in a hybrid simulation. 
rotational temperature along a line inclined $60^{\circ}$ from the freestream direction. Figure 10 (right) shows the corresponding profiles for bulk velocity and mass density. In both figures, small but noticeable differences are found between the two solutions in the post-shock region, with particularly significant discrepancies in the temperatures.

In the simulations described above, standard DSMC and LD-DSMC hybrid calculations are performed on the same mesh, in an effort to assess the accuracy of the hybrid scheme for an axisymmetric hypersonic blunt body flow. The simulations are performed in parallel on 12 processors in a cluster at the University of Michigan. The LDDSMC hybrid method does not show any computation expense reduction over simulations performed using standard DSMC. Total simulation times are approximately 394 hours for the standard DSMC and 660 hours for the hybrid LD-DSMC simulation (Table 3). To reduce the computational expense, an additional hybrid simulation is performed on a coarser mesh that provides grid independence in LD calculations, but is considerably less refined than necessary to meet DSMC guidelines. ${ }^{4}$ During collision selection routines as part of DSMC calculations, each cell in the DSMC domain is divided into a number of subcells, with subcell dimensions set to approximately half the local mean free path. LD-DSMC results (stream lines, Mach number and LD/DSMC domain boundaries) obtained with the coarser mesh (lower half) are compared to the full-mesh LD-DSMC results in Figure 5 (right). The coarser mesh uses a total of 91,342 cells. As expected, the shock is again captured in the DSMC domain and no significant differences are found in the location of continuum breakdown boundaries. In Figures 6 and 7, the LD-DSMC hybrid simulation results obtained with the coarse mesh are displayed as dashed lines. For the pressure, shown in Figure 5, very good agreement is observed between the coarse LD-DSMC results and results from the other two simulations. There is also very good agreement obtained with standard DSMC results for the translational and rotational temperature contours, as shown in Figure 7. The coarse mesh LD-DSMC profiles are included in Figures 8, 9 and 10 and are found to display the same general trends as LD-DSMC results obtained on the dense mesh. Vertical dotted lines in these figures denote the LD/DSMC domain boundaries obtained with the coarse mesh, and indicate slightly wider DSMC regions around both the shock and the boundary layer. The computational cost of the LD-DSMC simulation using the coarse mesh is about 392 hours; this is slightly less than the cost of the DSMC simulation, and considerably less than the hybrid calculation involving the dense mesh.

Table 3. Computation times

\begin{tabular}{cccc}
\hline \multicolumn{4}{c}{ Kn $=\mathbf{0 . 0 0 2}, \mathbf{M a}=\mathbf{1 0}$} \\
\hline Ttandard DSMC & LD-DSMC-1 & LD-DSMC-2 \\
\hline Notal of cell & 174,634 & 174,634 & 91,342 \\
Total CPU hours & 12 & 12 & 12 \\
\hline
\end{tabular}

Accurate prediction of surface properties is often the most important aspect of these types of computations for hypersonic vehicle design. Figures 11 and 12 show profiles along the sphere surface in pressure and heat flux, respectively. The pressure profiles obtained with DSMC and the LD-DSMC hybrid method (on both meshes) show excellent agreement while the heat flux results contain significant scatter near the stagnation point. To reduce the statistical noise, the stagnation point heat flux is averaged over the first ten cells along the surface. ${ }^{16,17}$ The stagnation point heat transfer coefficient and forebody total drag coefficient are calculated according to Equations (4) and (5). The values of the coefficients are listed in Table 4, where it is shown that the present LD-DSMC results agree with standard DSMC results to within $1 \%$.

Table 4. $C_{H}$ at the stagnation point, and $C_{D}$ over the forebody surface

\begin{tabular}{cccccc}
\hline \multicolumn{5}{c}{ Kn = 0.002, Ma = 10 } \\
\hline & Standard DSMC & LD-DSMC & Error & $\begin{array}{c}\text { LD-DSMC } \\
\text { (coarse mesh) }\end{array}$ & Error \\
$\mathbf{C}_{\mathbf{H}}$ & 0.0937 & 0.0940 & $0.3 \%$ & 0.0943 & $0.6 \%$ \\
$\mathbf{C}_{\mathbf{D}}$ & 0.9714 & 0.9714 & $0 \%$ & 0.9731 & $0.2 \%$ \\
\hline
\end{tabular}

$$
\begin{aligned}
& C_{D}=\frac{2 D}{\rho V_{\infty}^{2} S} \\
& C_{H}=\frac{2 q}{\rho V_{\infty}^{3}}
\end{aligned}
$$




\section{Conclusions and Future Work}

A hybrid particle scheme for the simulation of multiscale hypersonic flow has been described and assessed through comparison with DSMC simulation results. In the hybrid scheme, the DSMC method is used in rarefied regions and a low diffusion (LD) particle method is employed in continuum regions. The LD method has been shown to reduce numerical diffusion effects and scatter in comparison with other DSMC-based continuum particle methods, and is relatively simple to implement in a DSMC code for strongly coupled hybrid simulation of flows involving a wide range of Knudsen number regimes. Mach 10, $\mathrm{Kn}=0.002$ nitrogen flow over a sphere was used as a test case to evaluate the hybrid LD-DSMC scheme through a comparison of DSMC and hybrid simulation results using the same mesh. The results obtained with the two methods have good overall agreement, although the LDDSMC hybrid simulation is more expensive on a mesh refined to DSMC requirements. In order to reduce the computational expense of hybrid LD-DSMC calculations, a coarser mesh is employed in an additional hybrid simulation of the same flow. The enlarged cells do not violate any underlying approximations in the LD method, and can satisfy DSMC guidelines through the use of sub cells. The LD-DSMC results obtained on the coarser mesh also have good agreement for flow and surface properties with the full DSMC computation on the dense mesh. The computational expense of the LD-DSMC hybrid simulation with the coarse mesh is reduced by a factor of 1.7 in comparison to the LD-DMC hybrid simulation involving the dense mesh. Although this still does not represent an improvement in cost compared with the standard DSMC, this result suggests that the computational expense may be further reduced by using a still coarser mesh in continuum regions for the LDDSMC hybrid computation.

In the future, further hybrid LD-DSMC computations will be performed using a much coarser mesh and variable time step intervals to reduce the computational expense. The LD-DSMC hybrid algorithm will also be used to analyze Mars entry capsule aerothermodynamics. Figure 13 shows the forebody continuum breakdown boundary of Mars Pathfinder at $65 \mathrm{~km}$ altitude (Table 1). This computation will be extended to a full body simulation including the wake region as well as consideration of chemical

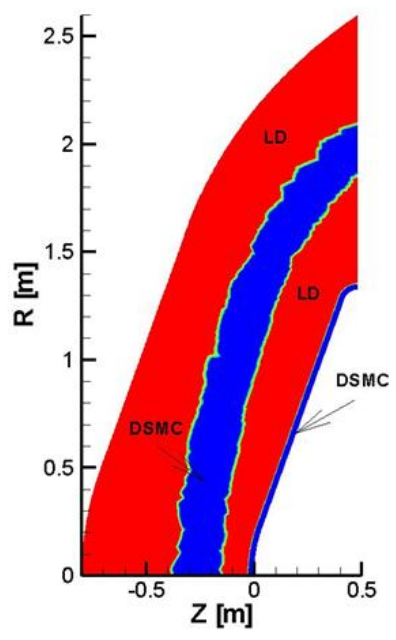

Figure 13. Continuum breakdown boundary for Mars pathfinder reactions.

\section{Acknowledgements}

The authors gratefully acknowledge the financial support provided by NASA grant NNX08AD02A.

\section{References}

${ }^{1}$ Hubbard, G. S., "The Exploration of Mars; Historical Context \& Current Results," American Institute of Aeronautics and Astronautics, 2004, AIAA 2004-3, 42nd AIAA Aerospace Sciences Meeting and Exhibit, Reno, NV, Jan.

${ }^{2}$ Moss, J. N., Wilmoth, R. G. and Price, J. M., "DSMC simulation of blunt body flows for Mars entries: Mars pathfinder and Mars microprobe capsules," American Institute of Aeronautics and Astronautics, 1997, AIAA 19972508, 32nd Thermophysics Conference, Atlanta, GA, June.

${ }^{3}$ Moss, J. N., Blanchard, R. C., Wilmouth, R. G. and Braun, R. D., "MARS pathfinder rarefied aerodynamics: computations and measurements," American Institute of Aeronautics and Astronautics, 1998, AIAA 1998-298, 36th Aerospace Sciences Meeting and Exhibit, Reno, NV, Jan.

${ }^{4}$ Bird, G. A., Molecular Gas Dynamics and the Direct Simulation of Gas Flows, Oxford University Press, 1994.

${ }^{5}$ M.S. Ivanov, S. F. G., "Computational hypersonic rarefied flows," Fluid mechanics, Vol. 30, 1998, pp. 469-505.

${ }^{6}$ Schwartzentruber, T. E. and Boyd, I. D., "A hybrid particle-continuum method applied to shock waves," Journal of computational physics, Vol. 225, 2007, pp. 1159-1174.

${ }^{7}$ Schwartzentruber, T. E., Scalabrin, L. C. and Boyd, I. D., "A modular particle-continuum numerical method for hypersonic non-equilibrium gas flows," Journal of computational physics, Vol. 215, 2006, pp. 402-416.

${ }^{8}$ Tiwari, S. and Klar, A., "An adaptive Domain Decomposition Procedure for Boltzmann and Euler Equations," Journal of Computational Applied Mathematics, Vol. 90, No.2, 1998, pp. 223-237.

${ }^{9}$ Macrossan, M. N., "A Particle-Only Hybrid Method For Near-Continuum Flows," Proceedings of the American Institute of Physics, Vol. 585, 2001, pp. 388-395. 
${ }^{10}$ Burt, J. M. and Boyd, I. D., "A low diffusion particle method for simulating compressible inviscid flows " Journal of computational physics, Vol. 227, 2008, pp. 4653-4670.

${ }^{11}$ Burt, J. M. and Boyd, I. D., "Extension of a Multiscale Particle Scheme to Near-Equilibrium Viscous Flows," American Institute of Aeronautics and Astronautics, 2008, AIAA 2008-3914, 40th Thermophysics Conference, Seattle, WA, June.

${ }^{12}$ Burt, J. M. and Boyd, I. D., "A hybrid particle approach for continuum and rarefied flow simulation," Journal of Computational Physics, Vol. 228, 2009, pp. 460-475.

${ }^{13}$ Burt, J. M. and Boyd, I. D., "Rotational and vibrational nonequilibrium in a low diffusion particle method for continuum flow simulation," American Institute of Aeronautics and Astronautics, 2009, AIAA 2009-3743, 41th Thermophysics Conference, San Antonio, TX, June.

${ }^{14}$ Boyd, I. D. and Chen, G., "Predicting failure of the continuum fluid equations in transitional hypersonic flows," American Institute of Physics, Vol. 7, No.1, 1994, pp. 210-219.

${ }^{15}$ Borgnakke, C. and Larsen, P. S., "Statistical collision model for monte carlo simulation of polyatomic gas mixture," Journal of computational physics, Vol. 18, No.3, 1975, pp. 405-420.

${ }^{16}$ Holman, T. D. and Boyd, I. D., "Effects of Continuum Breakdown on the Surface Properties of a Hypersonic Sphere," Journal of Thermophysics and Heat Transfer, Vol. 23, No. 4, 2009, pp. 660-673.

${ }^{17}$ Lofthouse, A. J. and Boyd, I. D., "Effects of continuum breakdown on hypersonic aerothermodynamics," Physics of fluid, Vol. 19, No. 027105, 2007, pp. 1-12. 

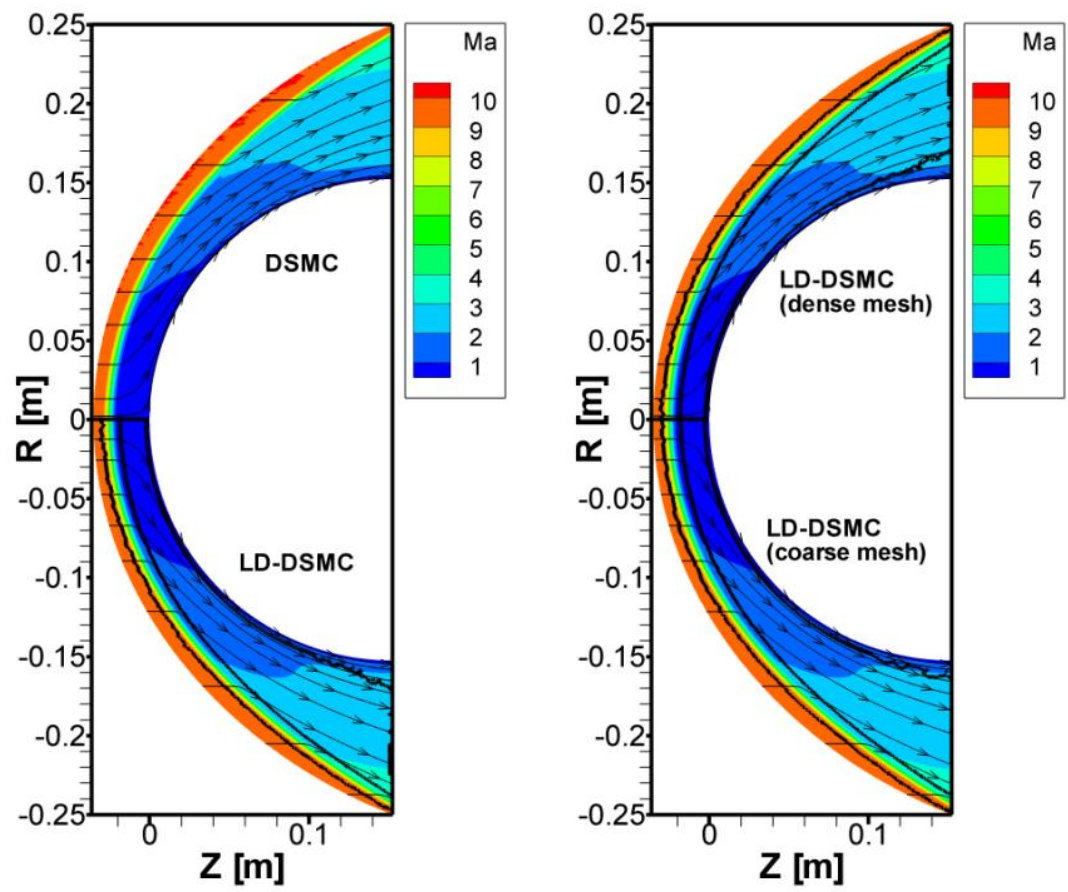

Figure 5. Stream lines, Mach number contours and LD, DSMC domain boundaries. (Left: upper $=$ DSMC, lower $=$ LD-DSMC using the same mesh; right: upper $=$ LD-DSMC with dense mesh, lower = LD-DSMC with the coarse mesh.)

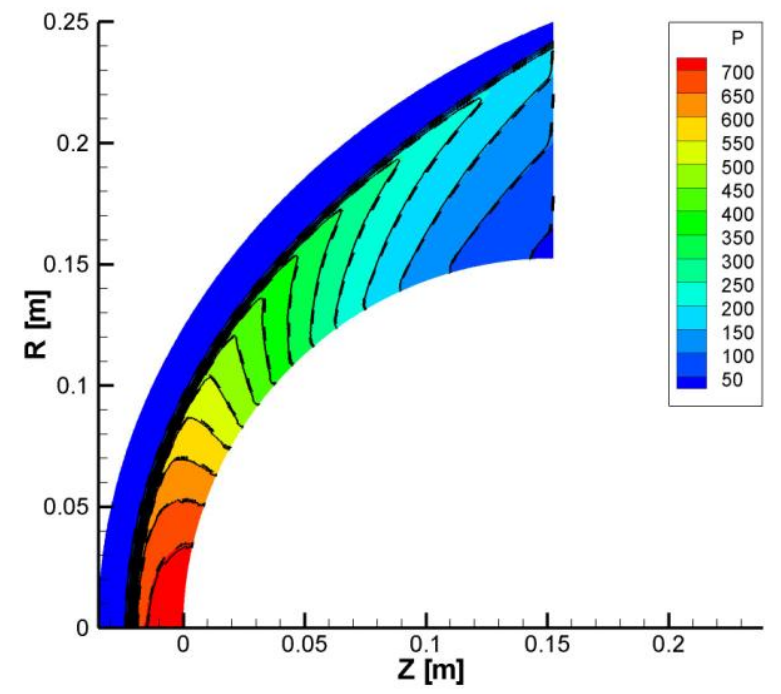

Figure 6. Pressure contours from DSMC and LD-DSMC hybrid simulations [Pa]. Contours and solid lines represent DSMC and LD-DSMC hybrid results, respectively, for simulations using the dense mesh. Dashed lines indicate LDDSMC hybrid results with the coarse mesh. 

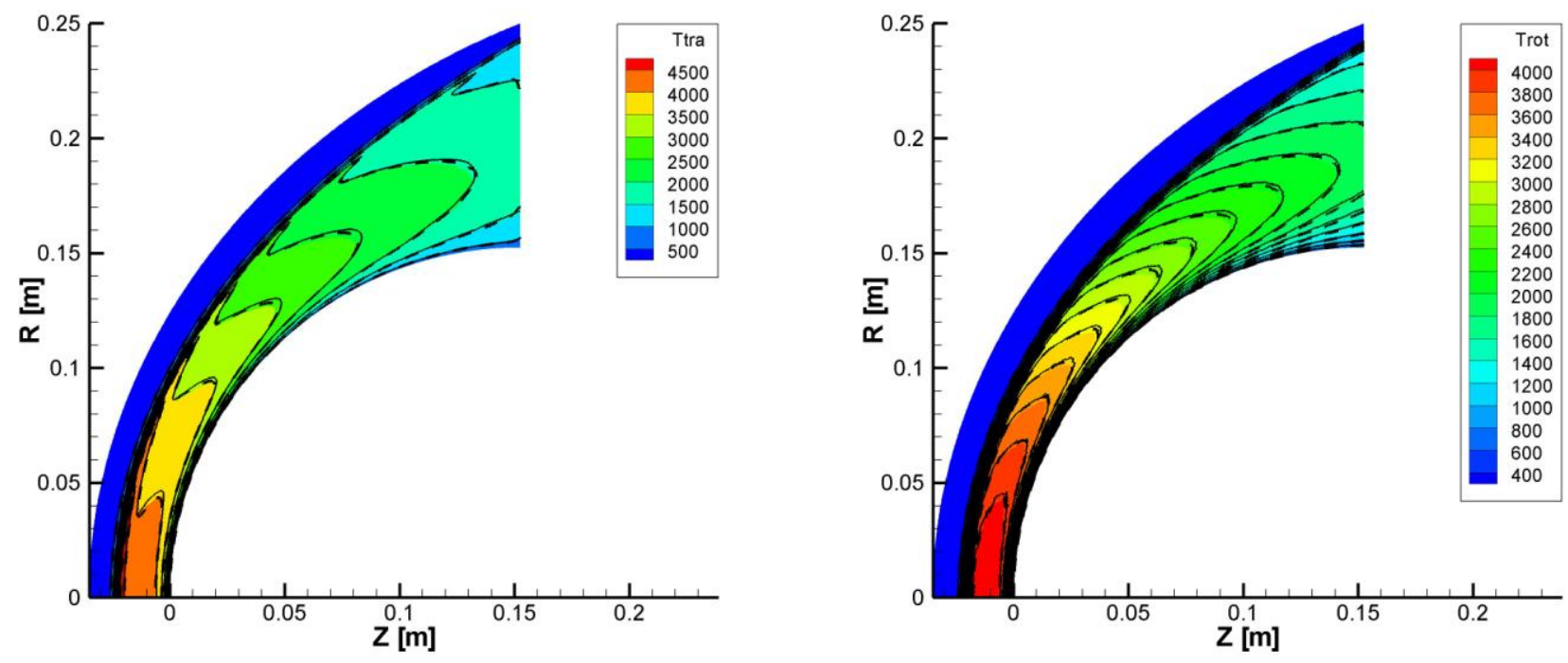

Figure 7. Contours of translational and rotational temperature [K]. Contours and solid lines represent DSMC and LDDSMC hybrid results, respectively, for simulations using the dense mesh. Dashed lines indicate LD-DSMC hybrid results with the coarse mesh.

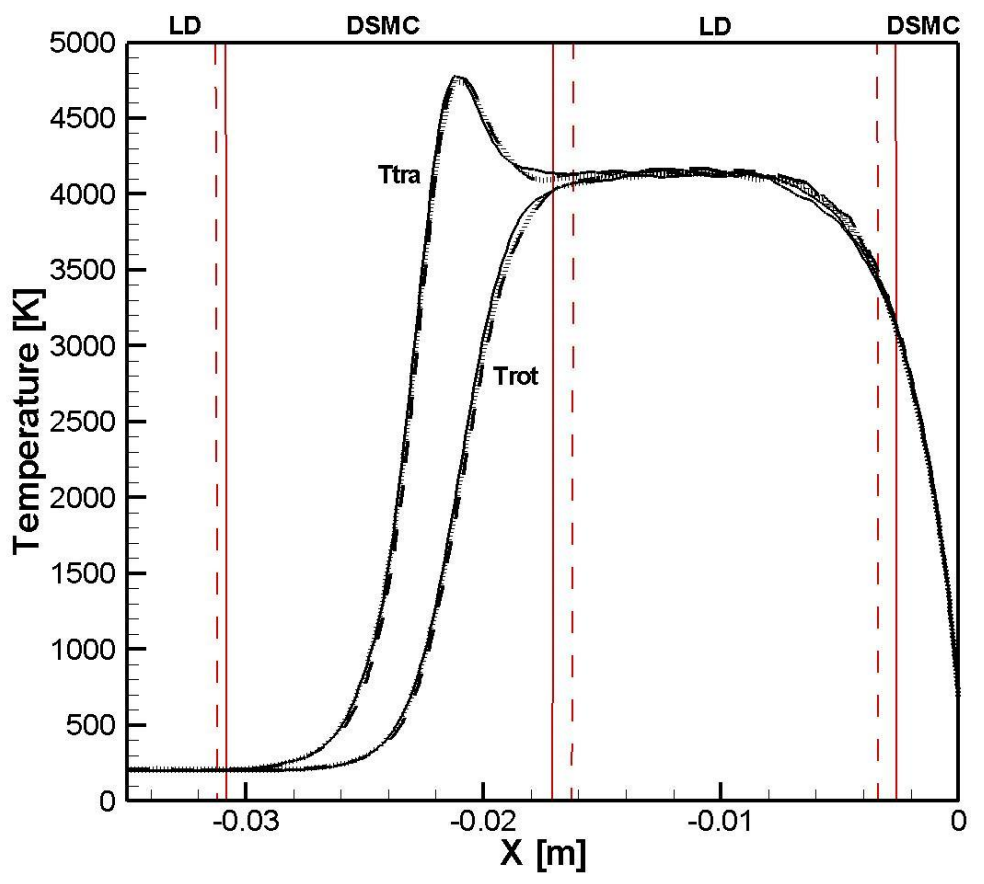

Figure 8. Profiles of translational and rotational temperature along the stagnation line. Solid lines and dashed lines represent DSMC and LD-DSMC hybrid results, respectively, for the dense mesh. Dotted lines denote LD-DSMC hybrid results for the coarse mesh 


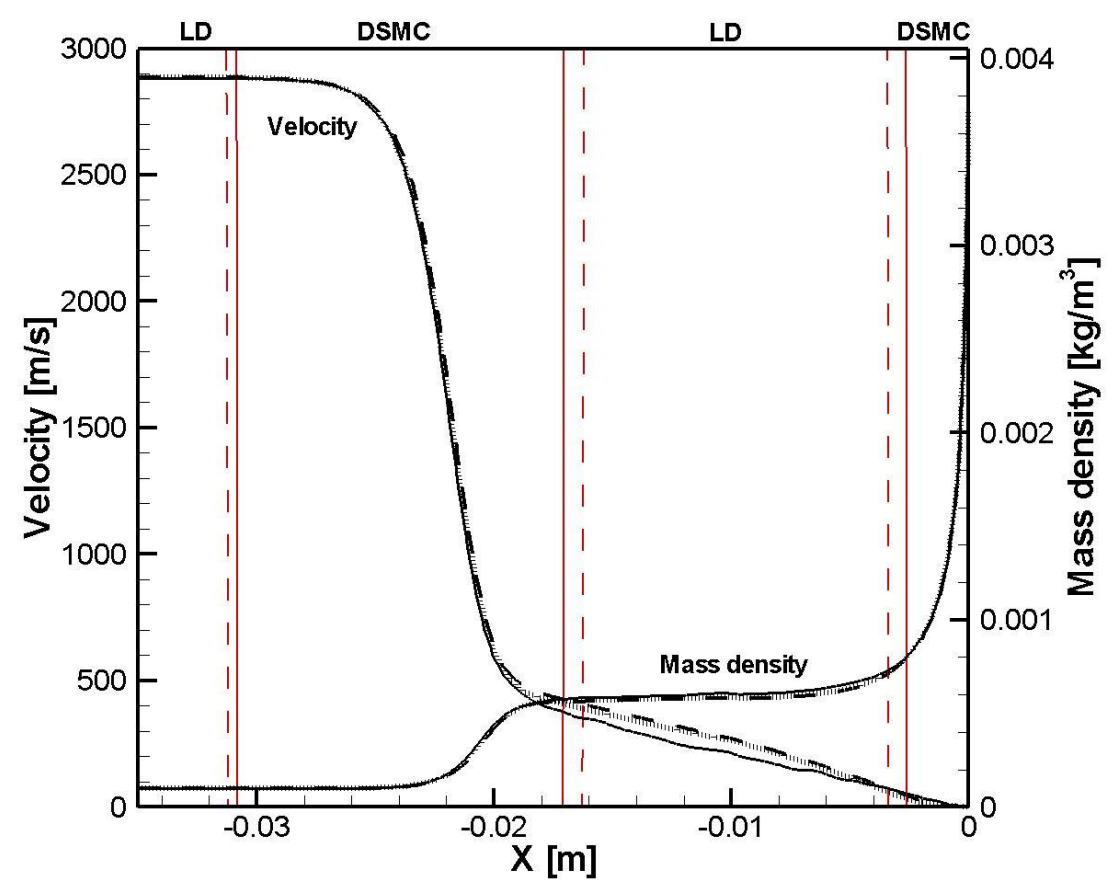

Figure 9. Profiles of bulk velocity and mass density along the stagnation line. Solid lines and dashed lines represent DSMC and LD-DSMC hybrid results, respectively, for the dense mesh. Dotted lines denote LD-DSMC hybrid results for the coarse mesh.
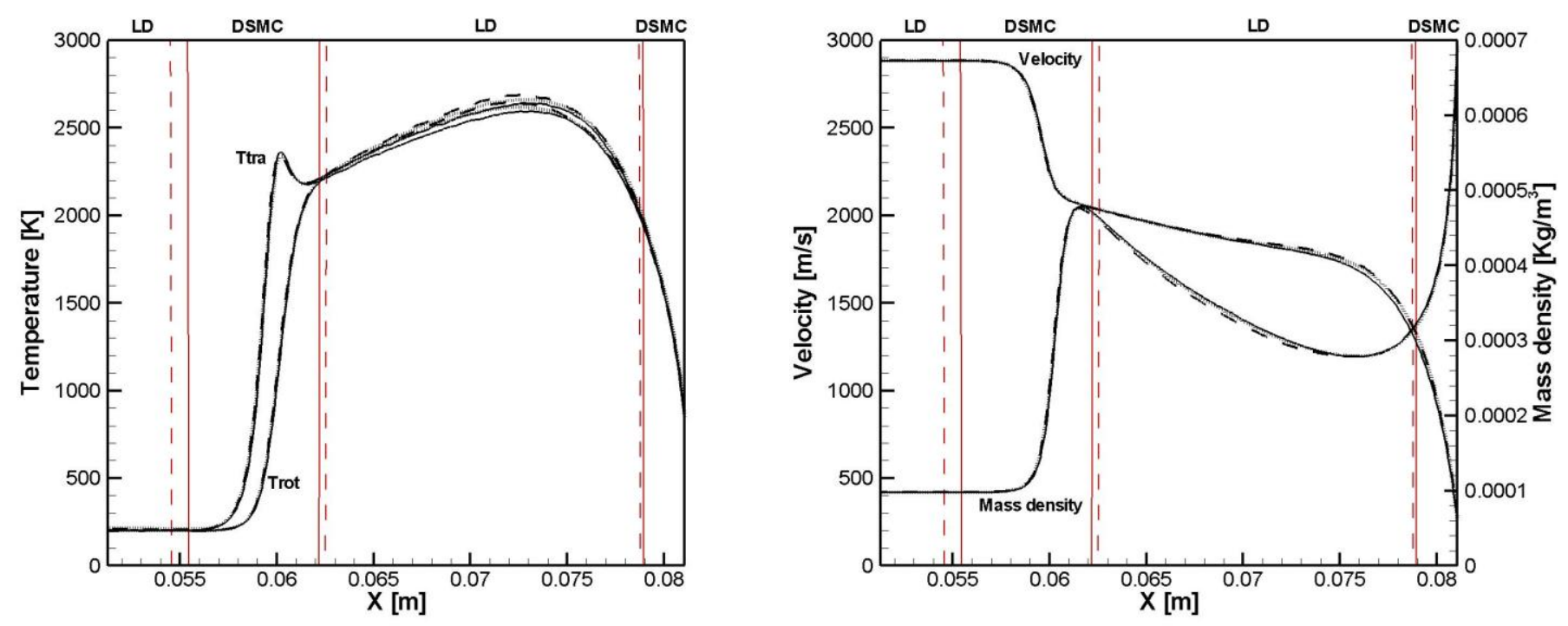

Figure 10. Profiles along an extraction line inclined $60^{\circ}$ from the freestream direction. (Left) Translational temperature, Rotational temperature (Right) Bulk velocity, Mass density. Solid lines and dashed lines represent DSMC and LD-DSMC hybrid results using the same dense mesh and dotted lines indicates LD-DSMC hybrid results with the coarse mesh 


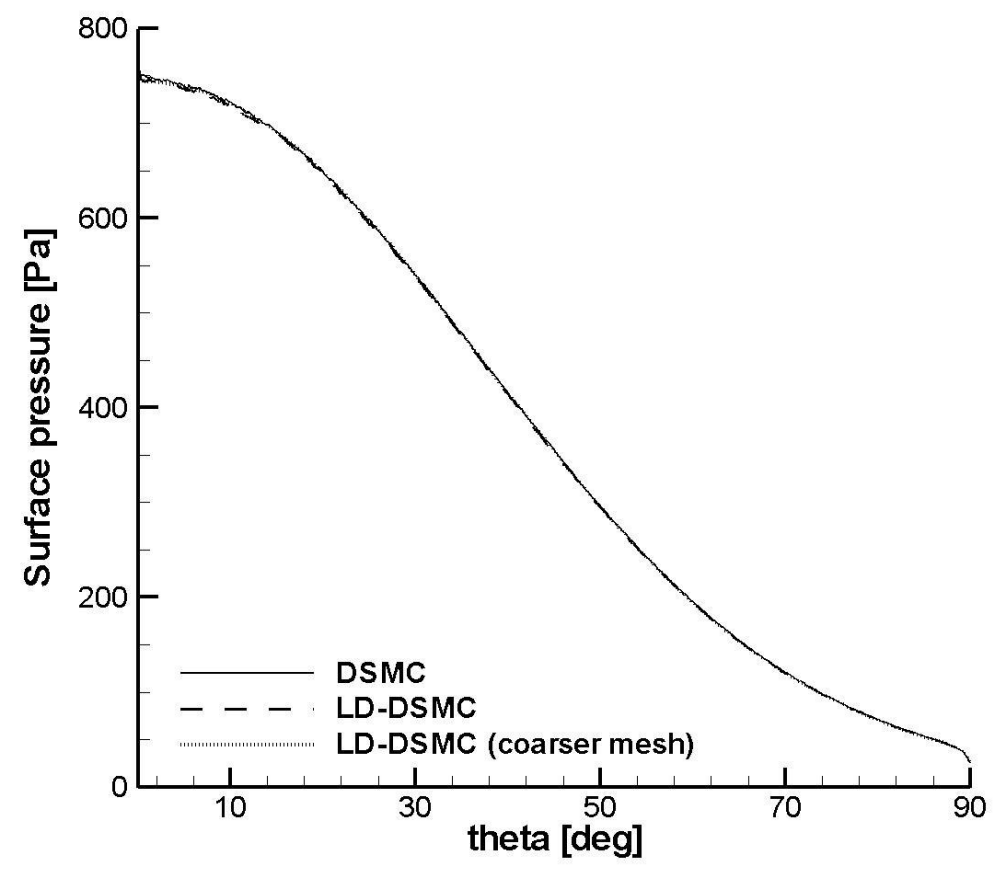

Figure 11. Surface pressure profiles.

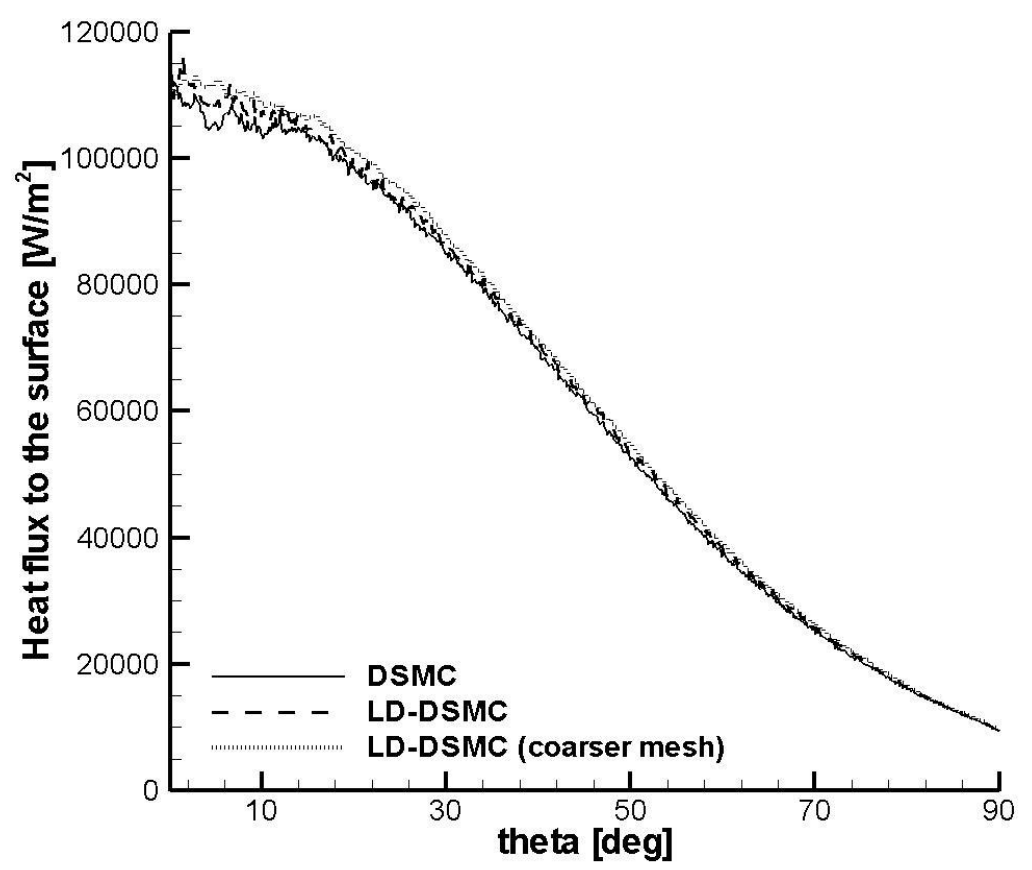

Figure 12. Surface heat flux profiles. 